\title{
On Some Characterization Results of Life Time Distributions using Mathai-Haubold Residual Entropy
}

\author{
Javid Gani Dar* and Bander Al-Zahrani** \\ *Department of Statistics, GDC (Boys), Anantnag, Kashmir India. \\ **Department of Statistics, Faculty of Sciences, King Abdulaziz University, Jeddah, Saudi Arabia.
}

\begin{abstract}
In the context of information theory, Shannon's entropy [16] plays an important role. In case, one has information about the current age of the component which can be taken into account for measuring its uncertainty, Shannon's entropy is not suitable as such. Consequently, Ebrahimi [3] proposed an alternative approach for characterization of distribution functions in terms of conditional Shannon's measure of uncertainty. In this paper, we propose generalized residual entropy function for characterization of some life time models. Also, upper and lower bound of hazard rate function in terms of generalized residual entropy have been obtained. Based on the proposed measure, we derive the generalized residual entropy function for some continuous lifetime models.
\end{abstract}

Key Words: Shannon;s entropy, Mathai-Haubold entropy, Residual entropy, Generalized residual entropy, Life time distribution.

AMS Subject Classification: 60E15, 62N05, 90B25, 94A17, 94A24

\section{Introduction:}

Let $X$ be a non- negative random variable denoting the life time of a system, a component or living organism with probability density function $f$, distribution function $F$ and reliability function $R$. It is assumed that the component is functioning at $t=0$ and it will fail to some $t>0$ so that $R(0)=1$. The basic characteristics of interest in the reliability theory are

$$
R(X)=P(X>x) \text {, the survival function, }
$$

$$
\begin{aligned}
& h(x)=\frac{f(x)}{R(x)}, \text { the hazard rate function and } \\
& r(x)=\frac{1}{R(t)} \int_{t}^{\infty} R(x) d x, \text { the mean residual life function. }
\end{aligned}
$$

Also the following relation can be easily obtained

$$
R(t)=\exp \left\{-\int_{0}^{t} h(x) d x\right\} .
$$

The following equation gives the functional relationship between the hazard rate function and mean residual life function

$$
h(t)=\frac{r^{\prime}(t)+1}{r(t)},
$$

where $r^{\prime}(t)$ is the derivative of $r(t)$ with respect to $t$.

Shannon [16] defined the basic measure of uncertainty associated with the random variable $X$ is given by

$$
H(X)=-\int_{0}^{\infty} f(x) \log f(x) d x
$$

The entropy is interpreted as the expected uncertainty contained in $f(x)$ about the predictability of an outcome of the random variable $X$.

Mathai- Haubold [7] introduced the generalized information measure

$$
M_{\alpha}(X)=\frac{1}{\alpha-1}\left[\int_{0}^{\infty} f^{2-\alpha}(x) d x-1\right], \alpha \neq 1,0<\alpha<2 .
$$

For various properties and applications of (1.2), one should refer to $[7,8]$

As $\alpha \rightarrow 1,(1.2)$ reduces to Shannon's information entropy given in (1.1).

As argued by Ebrahimi [2], if a unit is known to have survived up to an age $t$, then (1.1) is no longer useful in measuring the uncertainty about the remaining life time of the unit. The idea is that a unit with great uncertainty is less reliable than a unit with low uncertainty. Accordingly, he introduced a measure of uncertainty known as residual entropy for the residual life time distribution .The residual entropy of a continuous random variable $X$ is defined as 


$$
H(X ; t)=-\int_{t}^{\infty} \frac{f(x)}{R(t)} \log \frac{f(x)}{R(t)} d x
$$

where $R(t)$ is the reliability function of the random variable $X$. For $t=0,(1.3)$ reduces to (1.1).

\section{Some Characterization Results:}

Based on the measure defined in (1.2), we introduce the new kind of information measure that takes the current age of the system into consideration and generalizes (1.3) as $M_{\alpha}(X ; t)=\frac{1}{\alpha-1}\left[\frac{\int_{t}^{\infty} f^{2-\alpha}(x)}{R^{2-\alpha}(t)} d x-1\right], \alpha \neq 1,0<\alpha<2$

As $\alpha \rightarrow 1,(2.1)$ reduces to (1.3).

We now show that $M_{\alpha}(X ; t)$ uniquely determines the $R(t)$.

Theorem 2.1: $\quad$ Let $X$ be the non negative random variable having continuous density function $f$ and distribution function $F$ with survival function $R(t)$. Assume that $M_{\alpha}(X ; t)<\infty, \alpha \neq 1,0<\alpha<2$ and decreasing, then $M_{\alpha}(X ; t)$ uniquely determines $R(t)$.

Proof: From (2.1), we have

$$
\int_{t}^{\infty} f^{2-\alpha}(x) d x=R^{2-\alpha}(t)\left[(\alpha-1) M_{\alpha}(X ; t)+1\right]
$$

Differentiating above equation with respect to $t$, we get

$$
(2-\alpha) h(t)\left[(\alpha-1) M_{\alpha}(X ; t)+1\right]-h^{2-\alpha}(t)-(\alpha-1) M_{\alpha}^{\prime}(X ; t)=0
$$

where $h(t)=\frac{f(t)}{R(t)}$ is the hazard rate function.

Hence for fixed $t, h(t)$ is a solution of

$$
p(x)=(2-\alpha) x\left[(\alpha-1) M_{\alpha}(X ; t)+1\right]-x^{2-\alpha}-(\alpha-1) M_{\alpha}^{\prime}(X ; t)
$$

Differentiating both sides with respect to $x$, we have

$$
p^{\prime}(x)=(2-\alpha)\left[(\alpha-1) M_{\alpha}(X ; t)+1\right]-(2-\alpha) x^{1-\alpha} \text {. }
$$

For extreme value of (2.3), put $p^{\prime}(x)=0$, we have

$$
x_{t}=\left[1+(\alpha-1) M_{\alpha}(X ; t)\right]^{\frac{1}{1-\alpha}} .
$$

Also, $p^{\prime \prime}(x)=(\alpha-1)(2-\alpha) x^{-\alpha}$.

Two cases arises:

Case I: Let $\alpha>1$, then $p^{\prime \prime}(x)>0$. Thus $p(x)$ attains minimum at $x_{t}$. Also, $p(0)>0$ and $p(\infty)=-\infty$. Further, $p(x)$ decrease for $0<x<x_{t}$ and increases for $x>x_{t}$. So $x=h(t)$ is a unique solution to $p(x)=0$.

Case II: Let $0<\alpha<1$, then $p^{\prime \prime}(x)<0$. Thus $p(x)$ attains maximum at $x_{t}$. Also, $p(0)<0$ and $p(\infty)=\infty$. Further, $p(x)$ increase for $0<x<x_{t}$ and decrease for $x>x_{t}$. So $x=h(t)$ is a unique solution to $p(x)=0$.

Combining both the cases, we conclude that $M_{\alpha}(X ; t)$ uniquely determines $h(t)$, which uniquely determines $R(t)$.

Theorem 2.2: The uniform distribution over $(a, b), a<b$ can be characterized by decreasing generalized residual entropy.

Proof: Let $X \sim U(a, b), a<x<b$.

Therefore, $f(x)=\frac{1}{b-a}, a<x<b$

and $R_{X}(x)=\frac{b-x}{b-a}$.

Hence, $M_{\alpha}(X ; t)=\frac{1}{\alpha-1}\left[(b-t)^{\alpha-1}-1\right]$

and $\quad M_{\alpha}^{\prime}(X ; t)=-(b-t)^{\alpha-2}$.

Also, $x_{t}=\left[1+(\alpha-1) M_{\alpha}(X ; t)\right]^{\frac{1}{1-\alpha}}=(b-t)^{-1}$.

Thus, from (2.3) we have $p\left(x_{t}\right)=0$.

Hence the theorem is proved.

Theorem 2.3: The distribution of the random variable $X$ is exponential iff

$M_{\alpha}(X ; t)=k$, where $k$ is a constant.

Proof: Let $X \sim \exp (\theta)$.

Therefore,

$M_{\alpha}(X ; t)=\frac{\theta^{1-\alpha}}{(\alpha-1)(2-\alpha)}-\frac{1}{\alpha-1}=k$. 
Conversely, let $M_{\alpha}(X ; t)=k$.

Therefore $M_{\alpha}^{\prime}(X ; t)=0$.

Thus from (2.2), we have

$$
h(t)=[(2-\alpha)(\alpha c-c+1)]^{\frac{1}{1-\alpha}}=k, \text { a constant. }
$$

Hence $X \sim \exp (\theta)$.

Theorem 2.4: The relationship of the form

$$
M_{\alpha}^{\prime}(X ; t)=c h^{2-\alpha}(t)
$$

is satisfied iff the random variable $X$ have

(i) exponential distribution

for $c=0$.

(ii) Pareto II distribution with survival function

$$
R(t)=(1+p t)^{-q}, p>0, q>\frac{\alpha-1}{2-\alpha}, t>0
$$

for $c>0$ and

(iii) Beta (Mukherjee and Islam) distribution with survival function

$$
R(t)=(1-m t)^{n}, m>0, n>\frac{\alpha-1}{\alpha-2}, 0<t<\frac{1}{m}
$$

for $c<0$.

Proof: The proof of (i) is simple and hence omitted.

(ii) if $X$ has Pareto II distribution, then the p.d.f and hazard rate function is given by

$$
f(t)=p q(1+p t)^{-(q+1)} \text { and } h(t)=\frac{p q}{1+p t} \text { respectively. }
$$

Therefore

$$
M_{\alpha}(X ; t)=\frac{1}{\alpha-1}\left[\frac{(p q)^{2-\alpha}(1+p t)^{\alpha-1}}{p[(q+1)(2-\alpha)-1]}-1\right] .
$$

Differentiating above expression with respect to $t$, we have

$$
M_{\alpha}^{\prime}(X ; t)=c h^{2-\alpha}(t) \text {, }
$$

where $c=\frac{1}{(q+1)(2-\alpha)-1}>0$.

Conversely, let $M_{\alpha}^{\prime}(X ; t)=c h^{2-\alpha}(t), c>0$.

Therefore from (2.2), we have

$$
(2-\alpha) \int_{t}^{\infty} f^{2-\alpha}(x) d x=(\alpha c-c+1) h^{1-\alpha}(t) R^{2-\alpha}(t) .
$$

Differentiating both sides with respect to $t$, we get

$$
\frac{d}{d t}\left(\frac{1}{h(t)}\right)=c(2-\alpha)=k \text { (say). }
$$

Thus,

$$
h(t)=\frac{1}{k t+d}
$$

where $d>0$ is a constant.

Now if we let, $p=\frac{k}{d}, q=\frac{1}{k}$, then $h(t)=\frac{p q}{1+p t}$, which is the hazard rate function of Pareto II distribution.

(iii) Let $X$ follow beta distribution with p.d.f

$$
f(t)=m n(1-m t)^{n-1}, m, n>0,0<t<\frac{1}{m} .
$$

The hazard rate function is given by

$$
h(t)=\frac{m n}{1-m t} \text {. }
$$

The proof of first part follows from direct calculation.

For the converse part, $c<0$, the proof follows easily from (2.5).

Hooda and Kumar [6] characterize exponential distribution based on certain functional relation ship between the generalized residual entropy function and the mean residual function. In the next theorem, we characterize exponential distribution based on functional relation ship between the generalized residual entropy function defined in (2.1) and mean residual function,

THEOREM 2.5: Let $X$ be a continuous random variable, if $r_{\alpha}(t)$ and $M_{\alpha}(X ; t)$ be mean residual life function and generalized residual entropy function, where $r_{\alpha}(t)$ is defined as 


$$
r_{\alpha}(t)=\frac{1}{R^{2-\alpha}(t)} \int_{t}^{\infty} R^{2-\alpha}(x) d x, \alpha \neq 1,0<\alpha<2,
$$

then the relation given below

$$
M_{\alpha}(X ; t)+r_{\alpha}(t)=M_{\alpha}(X)+r_{\alpha}(0)
$$

holds for all $t \geq 0$ iff $X$ follows the exponential distribution.

Proof: Let $X \sim \exp (\theta)$.

Therefore,

$$
M_{\alpha}(X ; t)=\frac{\theta^{1-\alpha}}{(\alpha-1)(2-\alpha)}-\frac{1}{\alpha-1}
$$

and $r_{\alpha}(t)=\frac{1}{\theta(2-\alpha)}$.

Thus, $M_{\alpha}(X ; t)+r_{\alpha}(t)=M_{\alpha}(X)+r_{\alpha}(0)$.

Conversely, let (2.7) holds, we show that $X$ follows the exponential distribution.

Differentiating (2.7) with respect to $t$, we get

$$
M_{\alpha}^{\prime}(X ; t)+r_{\alpha}^{\prime}(t)=0
$$

Differentiating (2.6) with respect to $t$ and using the relation $h(t)=\frac{r^{\prime}(t)+1}{r(t)}$, we get

$$
r_{\alpha}^{\prime}(t)=(2-\alpha) r_{\alpha}(t) h(t)-1
$$

Substituting (2.9) and value of $M_{\alpha}^{\prime}(X ; t)$ from (2.2) into (2.8), we get

$$
h^{\prime}(t)\left[k-(2-\alpha) h^{1-\alpha}(t)\right]=0
$$

where $k=M_{\alpha}(X ; t)+r_{\alpha}(t)$ is a constant.

Thus, either $h^{\prime}(t)=0$ or $k-(2-\alpha) h^{1-\alpha}(t)=0$. Hence in both the cases $h(t)$ is constant. Hence the theorem is proved.

\section{New Class Of Life Time Distribution:}

In this section we give two new class of life time distribution based on generalized residual entropy $M_{\alpha}(X ; t)$. Based on this measure, we have the following definition:

Definition 3.1: A non negative random variable $X$ is said to have decreasing (increasing) uncertainty in generalized residual entropy DUGRL (IUGRL) if $M_{\alpha}(X ; t)$ is decreasing (increasing) in $t, t>0$.

This implies that the random variable $X$ has $D U G R L(I U G R L)$ if

$$
M_{\alpha}^{\prime}(X ; t) \leq(\geq) 0 \text {. }
$$

Theorem 3.1: If a distribution is $D U G R L$ as well as IUGRL for some constant $k$, then it must be exponential.

Proof: Since the random variable $X$ is both $D U G R L$ and IUGRL,

therefore,

$$
M_{\alpha}(X ; t)=k \text {, where } k \text { is a constant. }
$$

Differentiating both sides with respect to $t$, we get

$$
h(t)=[(2-\alpha)(\alpha c-c+1)]^{\frac{1}{1-\alpha}}=k \text {, a constant. }
$$

Therefore the distribution must be an exponential distribution.

The following theorem gives the upper and lower bound of $h(t)$ in terms of $M_{\alpha}(X ; t)$

Theorem 3.2: If $X$ is DUGRL (IUGRL), then

$$
h(t) \geq(\leq)\left\{(2-\alpha)\left[(\alpha-1) M_{\alpha}(X ; t)+1\right]\right\}^{\frac{1}{1-\alpha}} .
$$

Proof: The proof follows from Definition 3.1 and equation (2.2).

Corollary 3.1: If $X$ is $D U G R L(I U G R L)$, then

$$
R(t) \leq(\geq) \exp \left[-\int_{0}^{t}\left\{(2-\alpha)\left[(\alpha-1) M_{\alpha}(X ; u)+1\right]\right\}^{\frac{1}{1-\alpha}} d u\right] \forall t \geq 0 .
$$

\section{Generalized Residual Entropy Expressions for Some Life Time Models:}

Belzuence et. al [2] derive the residual entropy expression for some continuous distribution functions. Corresponding to these distributions, we derive the generalized residual entropy function based on the measure defined in (2.1).

(i) Exponential Distribution:

$$
\begin{aligned}
& f(x)=\theta e^{-\theta x}, x>0, \theta>0 . \\
& M_{\alpha}(X ; t)=\frac{\theta^{1-\alpha}}{(\alpha-1)(2-\alpha)}-\frac{1}{\alpha-1} .
\end{aligned}
$$

(ii) Uniform Distribution: 


$$
\begin{aligned}
& f(x)=\frac{1}{b-a}, a<x<b . \\
& M_{\alpha}(X ; t)=\frac{1}{\alpha-1}\left[(b-t)^{\alpha-1}-1\right] .
\end{aligned}
$$

(iii) Pareto II Distribution:

$$
\begin{aligned}
& f(x)=p q(1+p x)^{-(q+1)} . \\
& M_{\alpha}(X ; t)=\frac{1}{\alpha-1}\left[\frac{(p q)^{2-\alpha}(1+p t)^{\alpha-1}}{p[(q+1)(2-\alpha)-1]}-1\right] .
\end{aligned}
$$

(iv) Beta Distribution:

$$
\begin{aligned}
& f(x)=\theta x^{\theta-1}, 0<x<1, \theta>0 . \\
& M_{\alpha}(X ; t)=\frac{1}{\alpha-1}\left[\frac{\theta^{2-\alpha}\left(1-t^{(\theta-1)(2-\alpha)+1}\right)}{((\theta-1)(2-\alpha)+1)\left(1-t^{\theta}\right)^{2-\alpha}}-1\right] .
\end{aligned}
$$

(v) Finite Range Distribution:

$$
\begin{aligned}
& f(x)=\frac{\beta_{1}}{\vartheta}\left(1-\frac{x}{\vartheta}\right)^{\beta_{1}-1}, \beta_{1}>0,0 \leq t \leq \vartheta<\infty \\
& M_{\alpha}(X ; t)=\frac{1}{\alpha-1}\left[\frac{\beta_{1}^{2-\alpha}}{\vartheta^{1-\alpha}\left[\left(\beta_{1}-1\right)(2-\alpha)+1\right]}\left(1-\frac{t}{\vartheta}\right)^{\alpha-1}-1\right] .
\end{aligned}
$$

\section{References}

[1] Asaid, M and Ebrahimi, N (2000), "Residual entropy and its characterizations in terms of hazard function and mean residual life function”. Statist. Prob. Lett. 49: 263-269.

[2] Belzunce, F., Navarror, J., Ruiz, J.M and Aguila, Y (2004), "Some results on residual entropy function”.Metrika 59:147-161.

[3] Ebrahimi, N (1996), "How to measure uncertainty in the life time distributions".Sankhya. vol. 58, Ser.A , 48-57.

[4] Ebrahimi, N (1997), “Testing whether life time distribution is decreasing uncertainty”.J.Statist. Plann.Infer. 64:9-19

[5] Hooda, D. S and Saxena, S (2011), "Generalized measures of discrimination between past life time distributions", Pak.J.Stat. Oper. Res. Vol. VII, No. II,PP. 233-243.

[6] Hooda, D.S and Kumar, P (2007), "Generalized residual entropies in survival analysis", Journal of Applied Probability and Statistics, Vol. 2, No. 2, PP. 241-249.

[7] Mathai, A.M and Haubold, H. J (2006), “ Pathway models, Tsallis statistics, superstatistics and a generalized measure of entropy”, Physics A, Vol. 375, PP. 110-122.

[8] Mathai, A.M and Haubold, H. J (2006a), “ On generalized distributions and pathways”. arXiv:cond-mat/0609526v2.

[9] Mukherjee, S.P and Islam, A (1983), “A finite range distribution of failure times", Navel Res. Logist. Quat. Vol. 30, PP. 487-491.

[10] Nanda, A. K. and Gupta, R. D (2001), “ Some properties of reversed hazard rate function.” Statistical Methods, Vol. 3, PP. 108-124.

[11] Nanda, A. K. and Paul, P (2005), "Some properties of past entropy and their applications ." Metrika, Vol. 64, PP 47-61.

[12] Nanda, A. K. and Paul, P (2006), "Some results on generalized residual entropy.” Information Science, Vol. 176, PP. 24-47.

[13] Nair, K.R.M and Rajesh, G (1998), "Characterization of probability distributions using the residual entropy function".J.Indian Statist.Assoc. Vol. 36:157-166

[14] Renyi, A (1961), “On measure of entropy and information”.Proceeding of the Fourth Berkeley Symposium on Math.Statist.Prob.Vol 1, University of California Press, Berkely, 547-561.

[15] Sankaran, P.G. and Gupta, R.P (1999), "Characterization of the life time distributions using measure of uncertainty". Calcutta Statistical Association Bulletin. Vol. 49:154-166.

[16] Shannon, C.E (1948), “A mathematical theory of communication”. Bell System Technical J. Vol. 27:379-423 and 623-656.

[17] Varma, R.S (1966), "Generalizations of Renyi’s entropy of order $\alpha$ ”J.Math Sci. Vol. 1: 34-48 